\title{
Performance of an artificially aged module of the DELPHI HPC calorimeter
}

\author{
W. Bonivento, A. De Min ${ }^{1}$, S. Ragazzi, T. Tabarelli de Fatis, A. Tonazzo \\ Dipartimento di Fisica, Università di Milano, \\ and I.N.F.N. Sezione di Milano
}

\begin{abstract}
The readout proportional chambers of the HPC electromagnetic calorimeter in the DELPHI experiment are affected by large ageing. In order to study the calorimeter long-term behaviour, one HPC module was extracted from DELPHI in 1992 and was brought to a test area where it was artificially aged during a period of two years; an ageing level exceeding that expected for the HPC at the end of the LEP era was reached. During this period the performance of the module was periodically tested by means of dedicated beam tests whose results are discussed in this paper. These show that ageing has no significant effects on the response linearity and on the energy resolution for electromagnetic showers, once the analog response loss is compensated for by increasing the chamber gain through the anode voltage.
\end{abstract}

\footnotetext{
${ }^{1}$ Now at Dipartimento di Fisica, Università di Padova and I.N.F.N. Sezione di Padova
} 



\section{Introduction}

The readout proportional chambers of the HPC electromagnetic calorimeter in the DELPHI experiment are affected by large ageing, having its most evident manifestation in a progressive analog response loss. At present, the successful operation of the calorimeter is guaranteed by a procedure for precise monitoring of the pulse height evolution and amplitude loss compensation [1,2]. However, a careful verification of the reliability of this procedure on the long-term was deemed mandatory to ensure the detector operation up to the end of the LEP era [1]. In addition, the occurrence of several ageing side effects could in principle progressively deteriorate the detector performance in term of linearity and energy resolution.

In order to study the long-term behaviour of the calorimeter, one of the HPC modules was extracted from DELPHI and artificially aged during a period of two years. Its performance was periodically tested by means of dedicated beam tests. This paper is aimed at a detailed discussion both of the ageing programme pursued and of the results of the beam tests: the results quoted here supersede those presented in a preliminary form in [2].

\section{The HPC calorimeter in DELPHI}

The High-density Projection Chamber (HPC) is the barrel electromagnetic calorimeter of the DELPHI experiment at the LEP collider.

It is composed of 144 independent units (modules) arranged in a cylindrical structure around the beam interaction region. Each module, trapezoidal in shape, consists of a time projection chamber in which 41 thin layers of lead have been inserted to act as absorber material for electromagnetic showers. The total depth of the calorimeter amounts to $17.5 X_{0}$.

The ionization charge produced by electromagnetic showers in the $8 \mathrm{~mm}$ wide $\mathrm{Ar}-\mathrm{CH}_{4}$ (80-20\%) gas gaps between the lead walls is drifted by an electric field towards one end of the module, where it is collected by proportional counters and sampled at a frequency of $15 \mathrm{MHz}$. In each module the cathode of the proportional counters is segmented into 128 pads, which provide a readout granularity varying between 10 and $30 \mathrm{mrad}$ in azimuthal angle as a function of depth and nine samplings along the radial coordinate.

For fast triggering purposes, a plane of scintillators is inserted into one of the sampling gas gaps at a depth of $4.5 X_{0}$.

More detailed information on the HPC calorimeter construction and operation can be found in references $[2,3]$.

\section{Ageing in the HPC}

After the HPC was built, a specific ageing (relative amplitude loss in the chamber analog response per linear density of charge collected at the anode wire) in the readout chambers of order $10^{3} \mathrm{~cm} \cdot C^{-1}$ was observed. This large specific ageing, combined with the $\sim 10 \mathrm{pA} / \mathrm{cm}$ average current density drawn in the HPC chambers by the natural alpha activity of the lead converter, results in an analog response loss of order $10 \%$ per year of data taking (about $5 \times 10^{6} \mathrm{~s}$ ) $[1,2]$. 
This sizeable analog response loss is the most evident manifestation of ageing in the HPC and can be used to quantify the status of ageing of a module. Following the convention of ref. [1], in this paper use will be made of the ageing factor, defined as

$$
f_{a}(t)=\frac{\text { analog response of the new module }}{\text { analog response at time } t} .
$$

From this and the definition of specific ageing $\kappa$ one can easily derive the useful relation $f_{a}=\exp (\kappa \lambda)$, where $\lambda$ is the linear density of charge collected at the anode wire ${ }^{2}$.

At present, the amplitude loss in each module can be accurately monitored by means of a procedure especially developed for the HPC calorimeter, based on the use of radioactive gas $\left({ }^{83 m} \mathrm{Kr}\right)[1]$. The procedure consists in adding small quantities of ${ }^{83 m} \mathrm{Kr}$ gas to the gas mixture flowing in the HPC. The ${ }^{83 m} \mathrm{Kr}$ gas is emitted by a ${ }^{83} \mathrm{Rb}$ source which is placed in a by-pass of the gas supply circuit. The chamber analog response is monitored analyzing the signal produced by monoenergetic electrons from internal conversion in the ${ }^{83 m} \mathrm{Kr}$ decay to the ground state.

The amplitude loss is determined through monthly ${ }^{83 m} \mathrm{Kr}$ runs, with a typical uncertainty of a few percent in each module, and compensated for by applying software corrections in the offline data analysis (software equalization). In addition, once per year (usually at the beginning of the data taking period), the amplitude loss in each module is compensated for by increasing the chamber gain through the anode voltage (hardware equalization) $[1,2,4]$. The absolute energy scale of the calorimeter is fixed with reference to the energy deposited by Bhabha events $\left(e^{+} e^{-} \rightarrow e^{+} e^{-}\right)$, being the beam energy known with very good precision.

These software and hardware equalizations have ensured the successful operation of the HPC calorimeter since the startup of LEP in 1989.

However, side effects induced by ageing, such as worsening of the response linearity and of the energy resolution, rate dependence of the analog response, and even chamber breakdown, which are widely discussed in the literature [5], could in principle manifest themselves in the near future, affecting the detector performance in the LEP200 phase. In particular, the rate dependence of the analog response and its correlation with the age of the chambers was observed in HPC modules [1] and in HPC-like test chambers [6]; with the same chambers a clear correlation of the resolution $\sigma_{E} / E$ at the ${ }^{83 m} \mathrm{Kr}$ peak with ageing was also observed [6]. This evidence is reinforced by figure 1, which shows the correlation between the resolution $\sigma_{E} / E$ of the readout chambers at the ${ }^{83 m} \mathrm{Kr}$ peak and the ageing factor for the 144 HPC modules at the end of 1994 data taking. This could at least imply a progressive worsening of the precision on pulse height monitoring and chamber equalization ${ }^{3}$.

In order to evaluate the occurrence of these effects and to anticipate the detector performance in the years to come, a dedicated programme was pursued over the years 1993 and 1994 on a unit of the HPC. As mentioned in the introduction, the test module was forcedly aged to a level that represents what is expected for the HPC beyond the scheduled end of the LEP programme and its performance periodically monitored in dedicated beam tests at the CERN West Hall.

\footnotetext{
${ }^{2}$ In practice, this relation holds only if $\kappa$ is independent of the charge and the current density. This is true only to first order in the HPC [1].

${ }^{3}$ At present, the precision on the HPC monitoring is limited by the systematic uncertainties related to pressure and temperature variations and instabilities in the gas supply system [1].
} 


\section{The ageing programme on the test module}

During the LEP shutdown in the winter 1992/93, some HPC modules were replaced by spare ones or had the readout chambers rewired [7]. One of the most aged modules (denominated $\mathrm{C} 4$ ) was chosen as the most suitable for dedicated ageing studies.

When it was extracted from the HPC, the $\mathrm{C} 4$ module was characterized by an ageing factor $f_{a} \sim 6$. This large ageing factor, compared to an average of about 2 for the rest of the HPC at that time, was determined by the following factors:

- the module had been largely tested on electron beams in 1988, that is before the LEP startup;

- its readout chambers are affected by the intrinsically high specific ageing of $\sim 2.3 \times$ $10^{3} \mathrm{~cm} \cdot \mathrm{C}^{-1}$ compared to an average of $\sim 1.5 \times 10^{3} \mathrm{~cm} \cdot \mathrm{C}^{-1}$ for the other HPC modules;

- the current density drawn into its readout chambers by the alpha activity of the lead layers is about twice as much as the average current observed in the other HPC modules.

The large ageing factor observed in the $\mathrm{C} 4$ module at the end of 1992 is consistent with a rough estimate of about 4.5 based on its specific ageing and on a total linear density of charge of $0.65 \mathrm{mC} \cdot \mathrm{cm}^{-1}$ collected at the anode wire from 1990 to the end of 1992 . The charge collected before 1990 was not accurately monitored.

In the years 1993 and 1994 ageing was forced on the C4 module both by increasing the supply voltage of the readout chambers with respect to normal operation in DELPHI and by means of frequent calibrations with radioactive ${ }^{83 m} \mathrm{Kr}$. This determined a relative amplitude loss of about $1 \%$ per effective day ${ }^{4}$, compared to $\sim 0.2 \%$ per day for the average HPC module in normal operating conditions.

Figure 2 shows the ageing factor as a function of the total charge collected on the anode wires for the $\mathrm{C} 4$ module after it was extracted from DELPHI. At the end of the ageing programme an ageing factor $f_{a} \sim 16$ was reached and a total of $0.47 \mathrm{mC} / \mathrm{cm}$ was integrated over two years, corresponding to about 8 years of operation of an average HPC module in DELPHI. A fit of the law $f_{a}=\exp (\kappa \lambda)$ to the data points of the figure gives a specific ageing of $(2.3 \pm 0.1) \times 10^{3} \mathrm{~cm} \cdot \mathrm{C}^{-1}$. As indicated in the figure, the evolution of the performance of the module was monitored in four dedicated beam tests.

Before each beam test, a calibration with ${ }^{83 m} \mathrm{Kr}$ was performed to equalize the the $\mathrm{C} 4$ module to the same analog response of the HPC modules in DELPHI (hardware equalization). This was obtained at $1320,1360,1360$ and $1400 \mathrm{~V}$ anode voltage respectively ${ }^{5}$. For comparison, figure 3a shows the high voltage distribution which hardware equalized the HPC modules in DELPHI at the beginning of 1995. On the same figure, a conservative extrapolation of the high voltage distribution on the basis of the present HPC ageing level to the year 2000 is also shown. At the end of the century, only few modules in the tail of the distribution will be as much aged as the $\mathrm{C} 4$ module during the fourth beam test (BT4). This is better seen in figure 3b, which shows the number of HPC modules exceeding $f_{a}=16$ as function of the year. Two modules will have passed this limit in

\footnotetext{
${ }^{4}$ The effective time is the time with the HPC voltage on. In short, just time is used hereafter.

${ }^{5}$ An electron beam was used in the first two beam tests and a positron beam in the other ones.
} 
2000, nine modules in 2003 and half of the HPC in 2012. LEP is scheduled to operate until the end of 2000, thus the evolution of the $\mathrm{C} 4$ module performance can realistically anticipate the HPC behaviour at the end of the LEP era.

\section{The beam test setup}

The data were taken on the X7 beam line at CERN, which is a tertiary derivation of the SPS $450 \mathrm{GeV}$ proton beam. Electrons and positrons are produced by the interaction of the secondary beam (mainly pions) on a lead target. The momentum of the beam is selected by bending magnets, while four collimators define the momentum bite and the particle flux. The momentum dispersion, obtained by properly setting the collimators, was at the level of $2 \%$. This was monitored by means of a magnetic spectrometer consisting of four planes of multiwire chambers. The main backgrounds affecting the X7 beam consist of muons and pions; the relative contamination is limited to a few percent and increases with the beam energy.

The C4 module was installed in a dipole magnet providing a $0.9 \mathrm{~T}$ magnetic field, which is roughly $25 \%$ lower than the field intensity in DELPHI. The magnet can be either rotated in the horizontal plane or shifted perpendicularly to the beam line, in order to properly set the incidence angle and the distance of the beam impact point from the readout chambers. Inside the magnet the module was aligned with the drift field parallel to the magnetic field, by measuring the current induced in the readout chambers by the natural radioactivity of the lead as a function of the rotation angle of the module.

The position of the incident particles in the plane perpendicular to the beam axis was measured by a multiwire chamber (SFM), with $4 \mathrm{~mm}$ wire pitch, placed inside the magnet at a distance of $\sim 10 \mathrm{~cm}$ from the HPC module.

For the trigger, coincidence was required between a layer of scintillators placed directly on the beam line and the SFM chamber. For beam energies above $30 \mathrm{GeV}$ the coincidence with the signal from the HPC scintillator layer was also imposed: in this case a relatively high threshold was chosen for the photomultiplier output signal which reduced the contamination from muons and pions without significantly affecting the efficiency for electromagnetic showers.

The drift field intensity was set at $106 \mathrm{~V} / \mathrm{cm}$ which reproduces the HPC working conditions in DELPHI.

As mentioned above, before each beam test a hardware equalization of the module was performed.

\section{Offline analysis}

For the final analysis the events were selected on the basis of the following criteria:

i) The impact point measured by the SFM chamber was required to be within $1.5 \sigma$, where $\sigma$ is the beam spread in the plane perpendicular to the beam axis, from its mean value determined over the full run at each energy point.

ii) The particle momentum measured by the magnetic spectrometer was required to be within $3 \sigma$ from its mean value determined over the full run at each energy point. 
The reconstructed charge was then corrected for:

a) channel-to-channel disequalization, with coefficients determined from a dedicated ${ }^{83 m} \mathrm{Kr}$ calibration run [4],

b) pressure and temperature fluctuations,

c) attenuation along the drift [8],

d) beam momentum spread, on the basis of the information from the spectrometer. This improved the overall precision on the absolute momentum to better than $1 \%$.

e) time-dependent screening effects on anode wires. Details on such effects, related to the wire ageing, are reported in section 7.1.

The center of gravity of the reconstructed charge distribution was determined independently for each sampling layer and, in order to minimize the contribution of path-length fluctuations in the showers, only the charge collected within a distance of $\pm 7.5 \mathrm{~cm}$ from the center of gravity in the drift direction was considered.

In each run approximately 5000 events were collected, about $70 \%$ of which passed the fiducial cuts applied in the off-line analysis. Taking into account the energy resolution of the calorimeter, this sample provides a statistical accuracy much better than $1 \%$ on the average charge deposited by the shower and at the percent level on the resolution $\sigma_{Q} / Q$.

The systematic uncertainty affecting the off-line correction of the measured charge amounts to a few percent and is dominated by the uncertainty on the pressure and temperature corrections.

\section{Results of the beam tests}

\section{1 $\quad$ Screening effects}

In aged proportional counters, insulating material is progressively deposited on the electrodes. Under irradiation, a layer of charge is accumulated on this material, to an extent which depend on a precarious equilibrium of different parameters, and can locally affect the charge multiplication in the gas. One of the most striking manifestations of this phenomenon is the rate-dependence of analog response [5]. This effect was also observed in HPC modules when irradiated with high intensity electron beams [6].

This effect was seen on the $\mathrm{C} 4$ module during the beam tests. In this case the beam consisted of bunches of 50-200 electrons in two second bursts separated by ten second intervals. The observed pulse height reduction in the $\mathrm{C} 4$ chamber is shown in Figure 4. The effect was negligible for beam energies lower than $35 \mathrm{GeV}$; for higher energies, the amplitude decrease was between $2 \%$ and $9 \%$. The time interval between two bursts was enough for the wire to recuperate the full electric field.

It should be stressed that this effect is not relevant in DELPHI, where the event rate per module is much lower than in these beam tests. Moreover, the good linearity of the HPC for energies up to $50 \mathrm{GeV}$ (see section 7.2) ensures that the screening of the charge collected during a single shower is also negligible. 


\subsection{Linearity and energy resolution}

The linearity of response of the $\mathrm{C} 4$ module was studied by comparing the average measured charge with the nominal electron beam energy in the range from 2 to $100 \mathrm{GeV}$.

The average measured charge is plotted in figure 5, as a function of the beam energy for the last beam test (BT4), together with a straight line fit on the points from 2 to $50 \mathrm{GeV}$.

It has already been stressed that the age of the $\mathrm{C} 4$ module at the time the beam test BT4 was taken corresponds to the average HPC age expected for the year 2012, or, equivalently, to the age of the most aged module in the HPC expected for the year 2000 .

Figure 5 shows that no significant deviation from linearity is observed for energies up to $50 \mathrm{GeV}$. This guarantees that the method of using Bhabha events $\left(e^{+} e^{-} \rightarrow e^{+} e^{-}\right)$at $45 \mathrm{GeV}$ to obtain the absolute HPC calibration is reliable even with very aged chambers.

The deviations from linearity visible at higher energies is attributed to longitudinal leakage and gas gain saturation and are not induced by ageing, being observed in new modules as well.

Also shown in figure 5 is the energy resolution of the $\mathrm{C} 4$ module in BT4. The points corresponding to beam energies from 2 to $50 \mathrm{GeV}$ fit the law

$$
\frac{\sigma_{E}}{E}(\%)=\frac{28.7 \pm 0.3}{\sqrt{E(\mathrm{GeV})}} \oplus 2.2 \pm 0.4
$$

The effect of ageing on the HPC performance can be deduced from figure 6 and 7 , where the results of the four beam tests are compared in the linearity region. In particular, figure 6 shows the ratio of the average charge measured in the BT1, BT2 and BT3 beam tests to the one measured in BT4 as function of the beam energy. All ratios are compatible with unity. The spread of the experimental points is due both to the uncertainties on the offline corrections to the measured charge and to the precision of hardware equalizations: then this spread can also be interpreted as an upper limit to the precision of the hardware equalizations. The hardware equalizations appear therefore to be reliable in the recovery of the analog response loss even in the presence of large ageing and the precision of the equalization turns out to be of the same order as the one presently achieved in the HPC [1].

Finally figure 7 shows the ratio of the energy resolution measured in BT1, BT2, BT3 and in a beam test in 1988 (new module) to the that measured in the BT4 beam test as a function of beam energy. Again, good consistency with unity is found and no correlation of the resolution with the age of the module can be inferred. The somewhat better behaviour of the C4 module in 1988, particularly at low energies, could be ascribed to the higher operating gas gain at that time, resulting in a reduced importance of threshold effects ${ }^{6}$.

\section{Conclusions}

The expected behaviour of the DELPHI HPC calorimeter with very aged readout chambers was studied by artificially ageing one module (C4 module).

From our tests we conclude that the performance of the HPC in terms of linearity of response and energy resolution is not significantly degraded by ageing.

\footnotetext{
${ }^{6}$ The gas gain of the HPC has been reduced by more than a factor two with respect to its design value, in order to reduce the current density in the readout chambers and, as a consequence, the ageing $[1,2]$.
} 
Ageing seems to affect only the chamber analog response in amplitude, but this effect can be monitored with periodic ${ }^{83 m} \mathrm{Kr}$ calibration runs and can be compensated for by both software and hardware corrections.

\section{Acknowledgements}

We acknowledge all the people who were involved in activities connected with this paper and especially those from the DELPHI HPC collaboration who helped us during the data taking period. In addition we would like to thank L. Gatignon of the SL division for his help in the preparation of the beam test set up.

\section{References}

[1] A. Cattai et al., Monitoring of the HPC by means of Radioactive Gas Calibrations, DELPHI Internal Note 93-129 CAL 108 (1993).

[2] A.De Min et al., Performance of the HPC Calorimeter in DELPHI, CERN-PPE/95$04(1995)$.

[3] DELPHI Collaboration, The DELPHI detector at LEP, Nucl.Instr. and Meth. A303(1991)233.

[4] A. Cattai et al., Equalization of the Readout Channels of the HPC by means of Radioactive Gas, DELPHI Internal Note 93-115 CAL 105 (1993).

[5] J.Va'vra, Review of Wire Chamber Ageing, in Workshop on Radiation Damage to Wire Chambers, J.A.Kadyk ed., Berkeley, USA, 1986.

J.A.Kadyk, Wire Chamber Ageing, Nucl.Instr. and Meth. A300(1991)436.

[6] A.Algeri et al., Nucl.Instr. and Meth. A338(1994)348.

[7] A.Algeri et al., HPC intervention during the 1992/92 shutdown, DELPHI Internal Note 93-145 CAL 100.

[8] W.Bonivento et al., Performance of a HPC module operated with increased drift field, DELPHI Internal Note 95-3 CAL 125 (1995). 


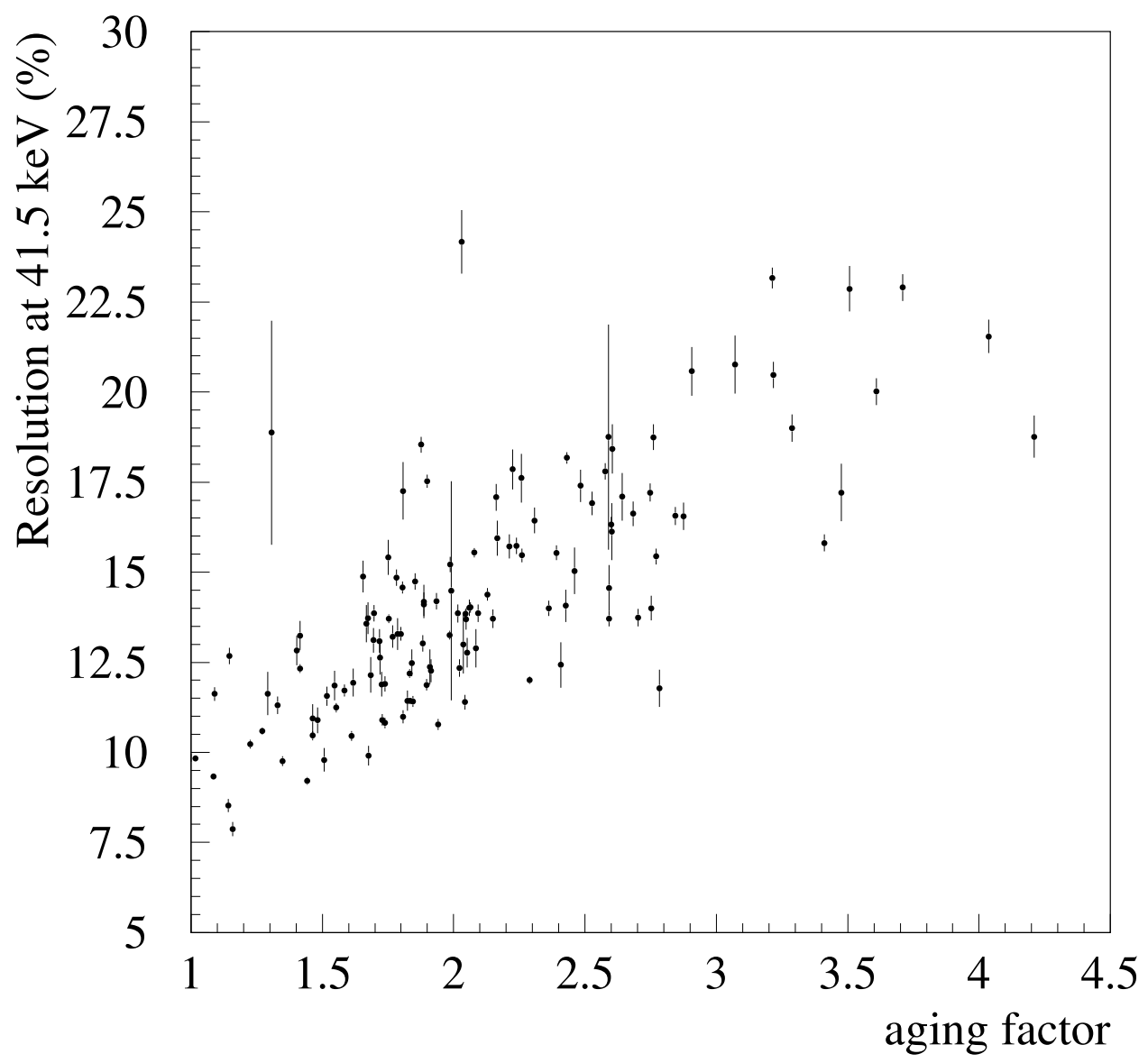

Figure 1: Resolution $\sigma_{E} / E$ at the ${ }^{83 m} \mathrm{Kr}$ peak versus the module ageing factor for the 144 HPC modules at the end of 1994 data taking. 


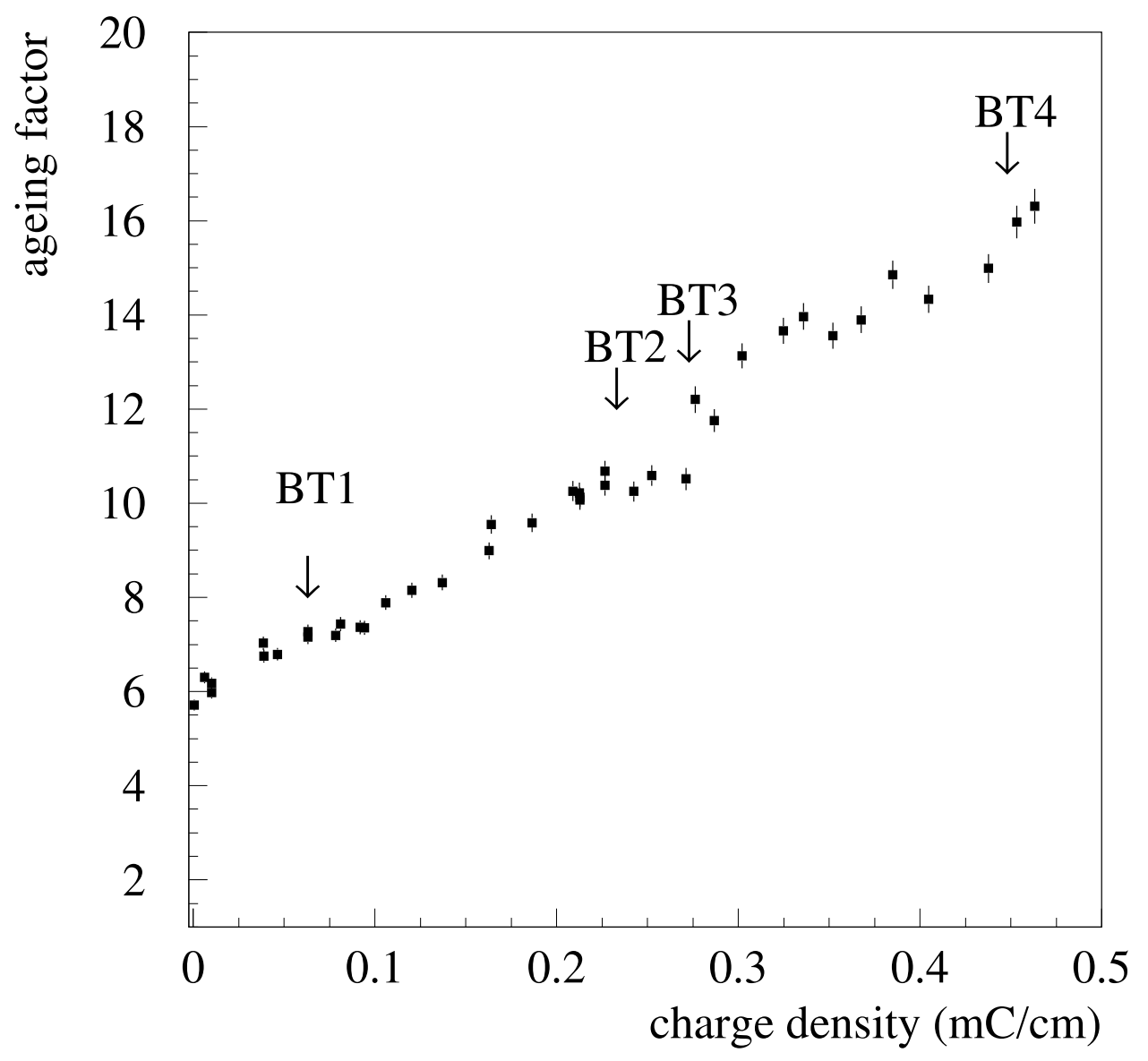

Figure 2: Ageing factor versus charge density collected at the wire for the HPC module under study after it was extracted from DELPHI: the arrows indicate the amount of charge integrated before each beam test. 

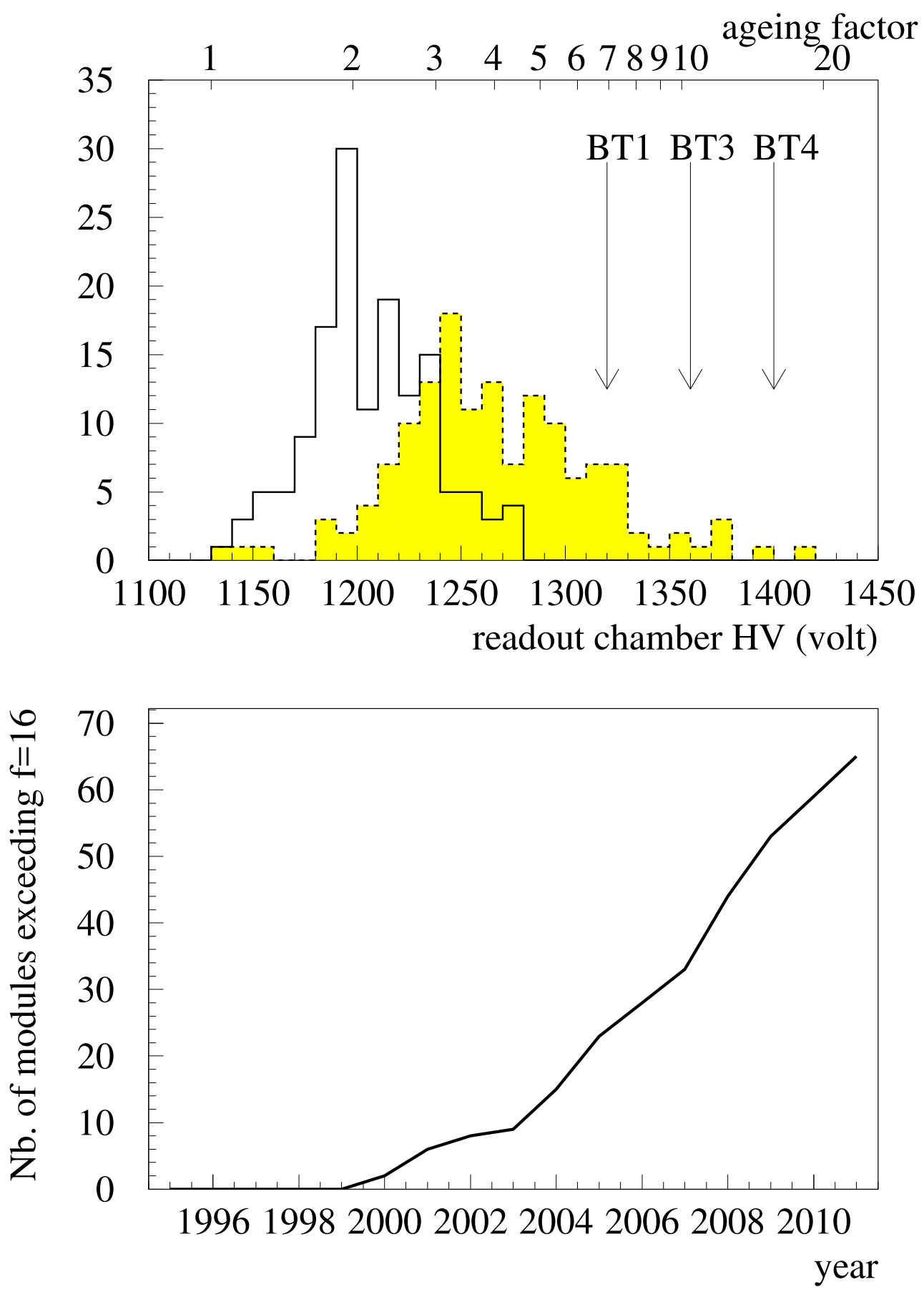

Figure 3: a) High voltage distribution for modules equalizations in 1995 (white histogram) and 2000 (grey histogram). The arrows indicates the anode high voltage of the $C_{4}$ module during the beam tests and corresponds to an ageing factor of 7, 10, 16 respectively; b) Number of HPC modules reaching an ageing factor greater than 16 as a function of the year. 


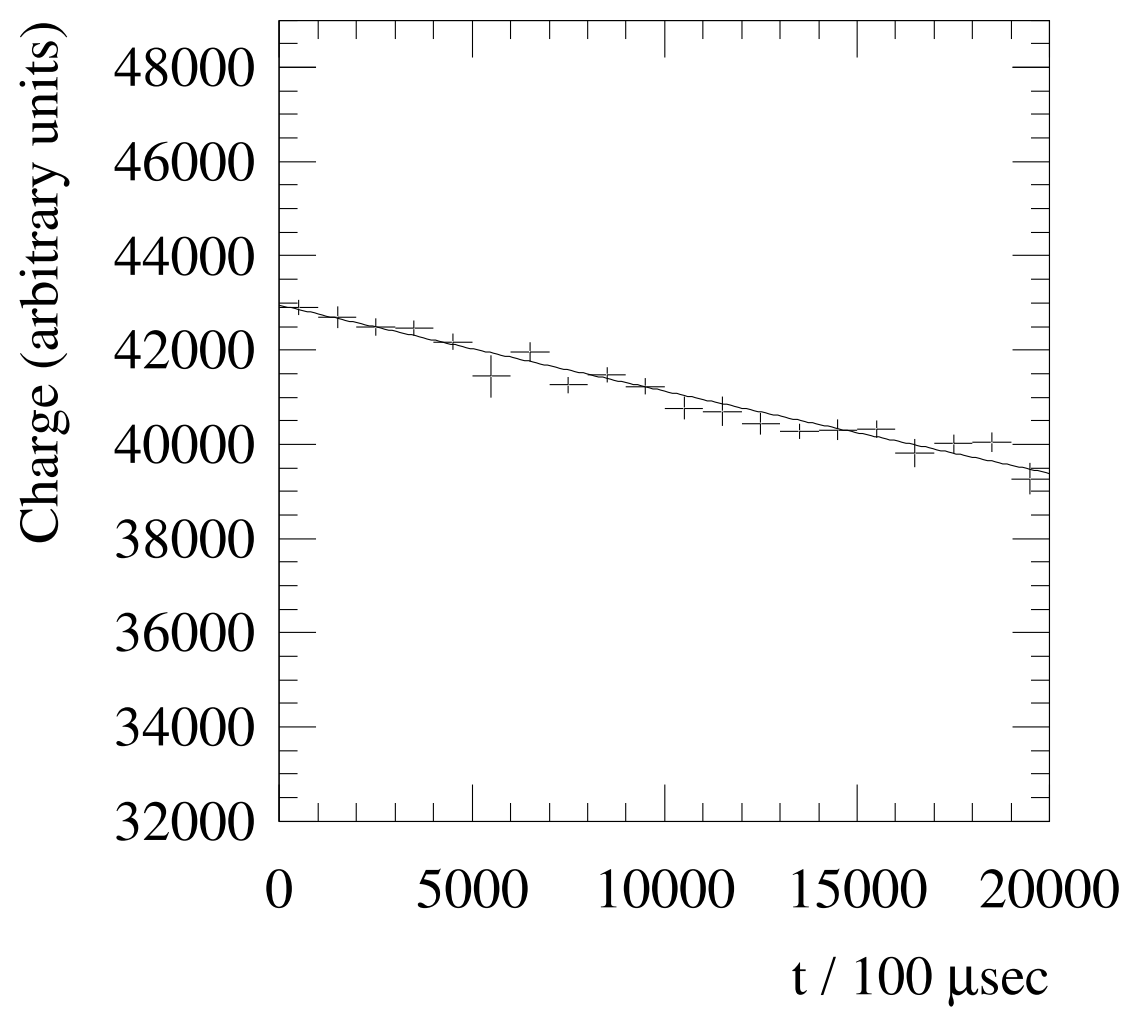

Figure 4: Average charge as a function of the time during the burst for a 50 GeV run in the BT1 beam test. The line is drawn to guide the eye. 

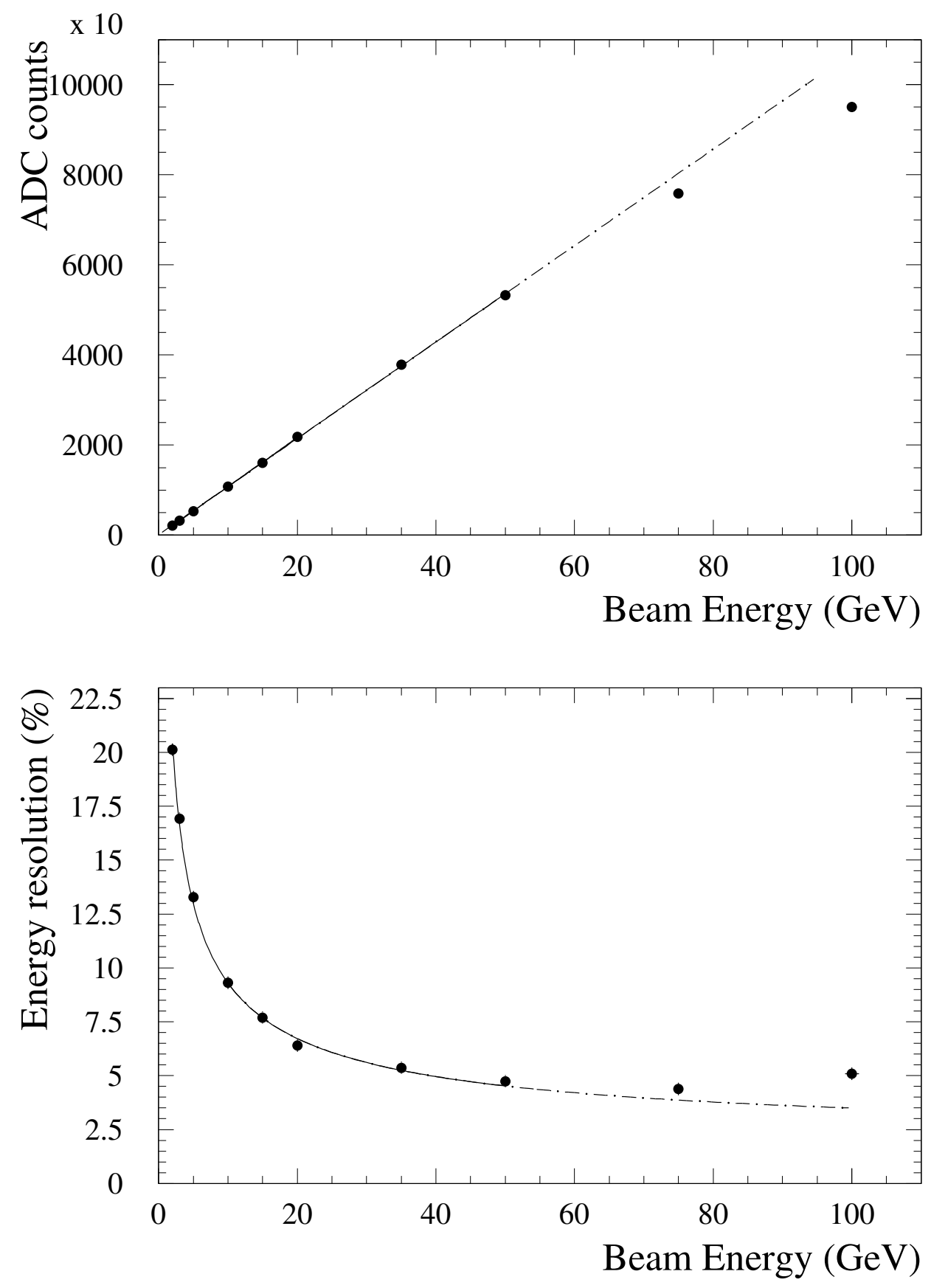

Figure 5: Upper plot: Average charge measured by the HPC module under test in the BT4 beam test versus beam energy: the result of a straight line fit including energy points up to $50 \mathrm{GeV}$ is also shown. Lower plot: Percent energy resolution measured by the HPC module under test in the BT\& beam test versus beam energy: the result of a fit including energy points up to $50 \mathrm{GeV}$ is also shown; see text for details. 


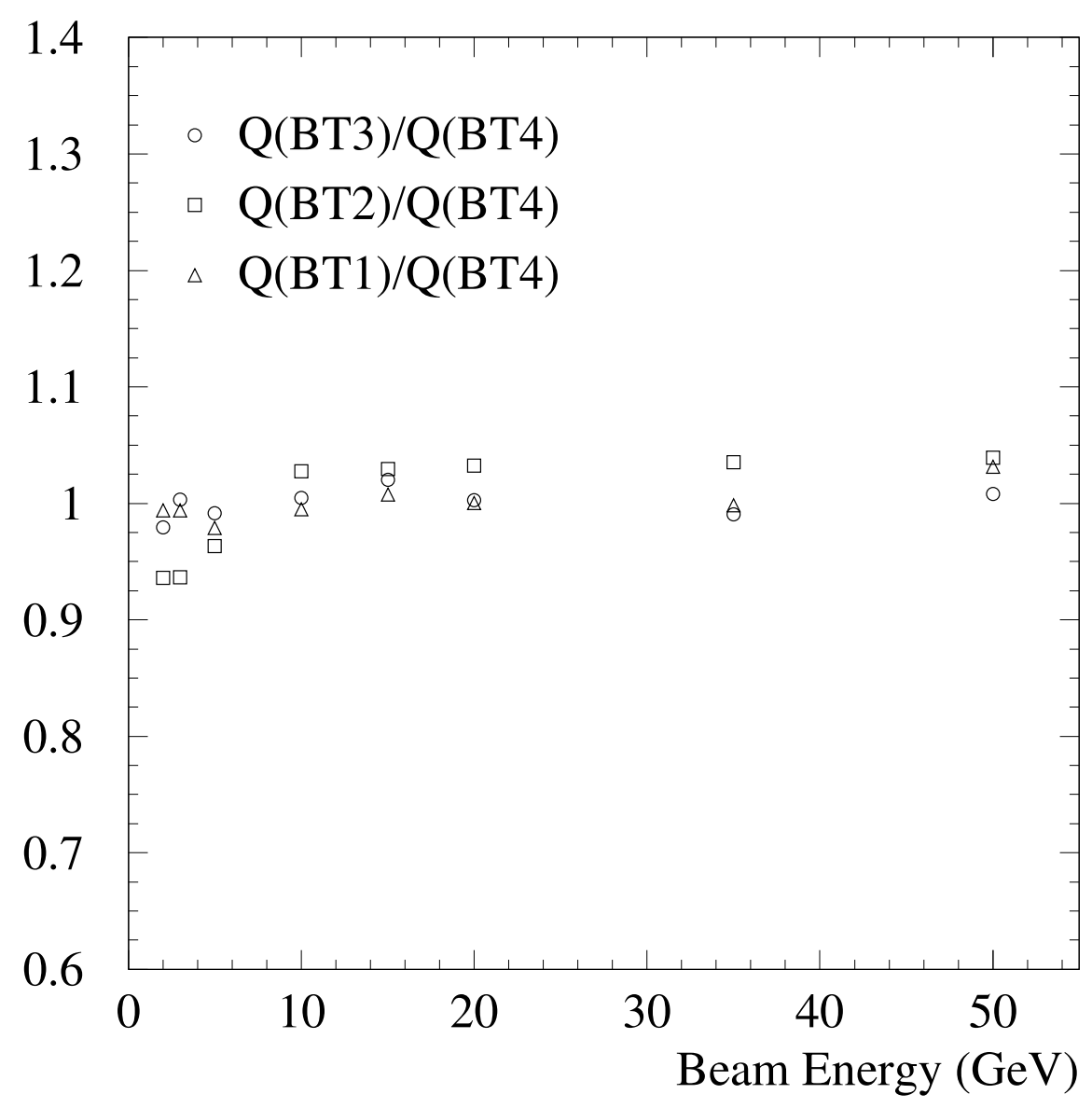

Figure 6: Ratio of average charge (Q) measured with the HPC module under test for the BT1,BT2 and BT3 beam tests to the one measured in the BT\& beam test as a function of beam energy. 


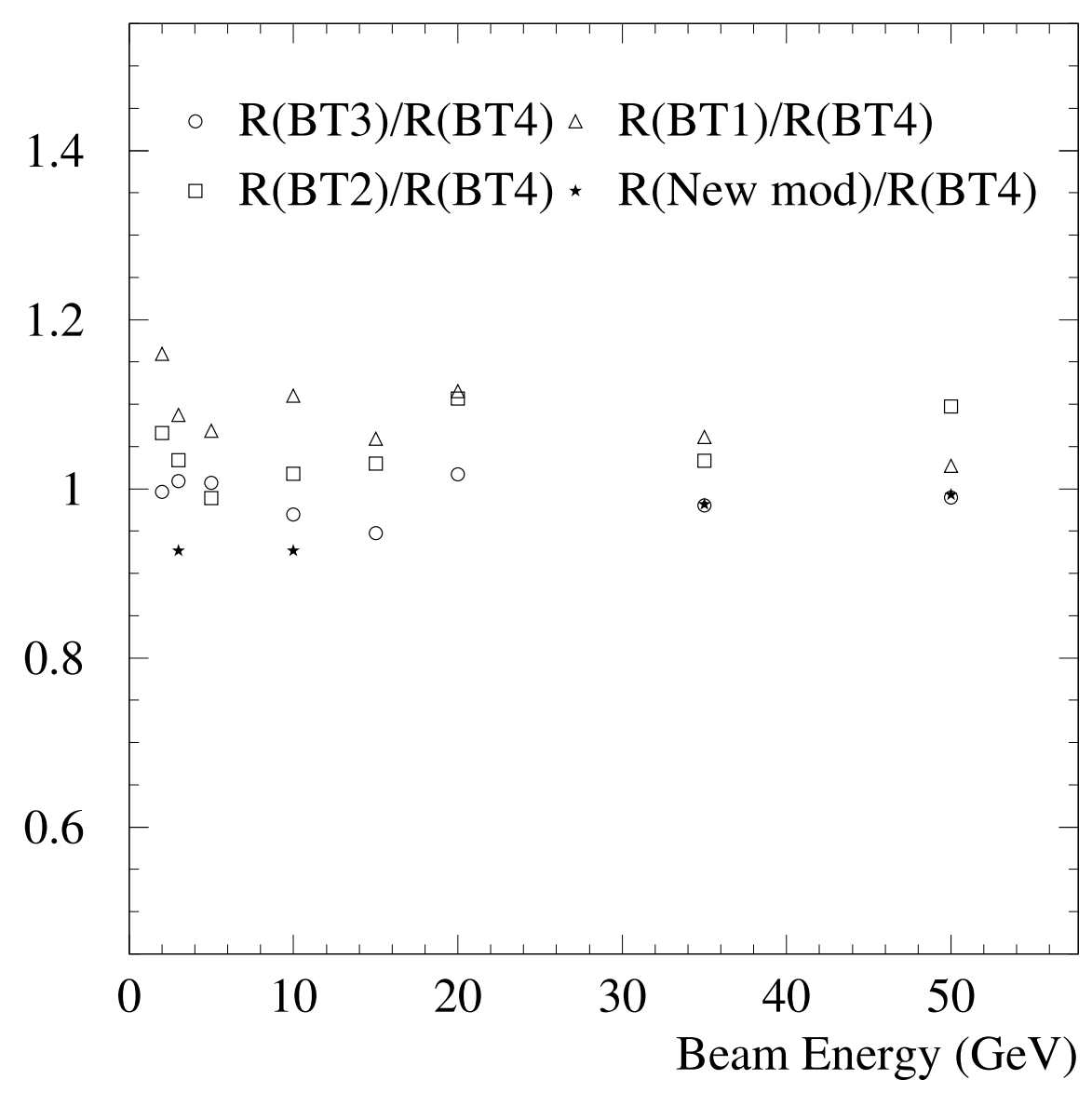

Figure 7: Ratio of the energy resolution $R=\sigma(E) / E$ measured in the BT1,BT2 and BT3 beam tests to the one measured in the BT4 beam test, as a function of beam energy. A comparison with the resolution of a new module, measured in a 1988 beam test, is also shown. 